\title{
Adolescents and Young Adults With a "Rare" Cancer: Getting Past Semantics to Optimal Care for Patients With Germ Cell Tumors
}

\author{
Sara J. Stoneham, ${ }^{a}$ Juliet P. Hale, ${ }^{b}$ Carlos Rodriguez-Galindo, ${ }^{c}$ Ha Dang, ${ }^{d}$ Thomas Olson, ${ }^{e}$ Matthew Murray,

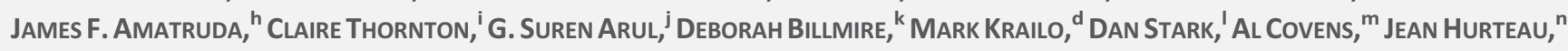 \\ Sally Stenning, ${ }^{\circ}$ James C. Nicholson, ${ }^{f}$ David Gershenson, ${ }^{\text {h }}$ A. Lindsay Frazier ${ }^{c}$ \\ ${ }^{a}$ Children's and Young Persons Cancer Services, University College London Hospital Trusts, London, United Kingdom; ${ }^{b}$ Department of \\ Paediatric Oncology, Royal Victoria Infirmary, Newcastle upon Tyne Hospitals trust, Newcastle upon Tyne, United Kingdom; 'Department of \\ Pediatric Oncology, Dana-Farber Cancer Institute and Boston Children's Hospital, Boston, Massachusetts, USA; ${ }^{\mathrm{d} C h i l d r e n ' s ~ O n c o l o g y}$ \\ Group, Monrovia, California, USA; ${ }^{\mathrm{e}}$ Department of Pediatric Oncology, Children's Healthcare of Atlanta, Emory University, Atlanta, Georgia, \\ USA; 'Department of Paediatric Oncology, Cambridge University Hospitals NHS Foundation Trust, Cambridge, United Kingdom;

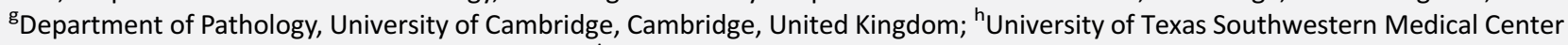 \\ and Children's Medical Center, Dallas, Texas, USA; 'Department of Pathology, Royal Victoria Hospital, Belfast Health Trust, Belfast, \\ United Kingdom; 'Department of Pediatric Surgery, Birmingham Children's Hospital NHS Foundation Trust, Birmingham, United Kingdom; \\ ${ }^{k}$ Riley Hospital for Children, Indianapolis, Indiana, USA; 'Department of Medical Oncology, St. James's Institute of Oncology, St. James \\ University Hospital, Leeds, United Kingdom; ${ }^{\mathrm{m}}$ Department of Gynecology Oncology, University of Toronto, Sunnybrook Health Sciences \\ Center, Ontario, Canada; ${ }^{n}$ Department of Gynecologic Oncology, North Shore University Health System, Evanston, Illinois, USA; ${ }^{\circ}$ Medical \\ Research Council Clinical Trials Unit, London, United Kingdom \\ Disclosures of potential conflicts of interest may be found at the end of this article.
}

Key Words. Adolescence - Young adults - Germ cell tumors • Risk stratification

\section{INTRODUCTION}

Germ cell tumors (GCTs) are the third most common cancer diagnosis in adolescent and young adult (AYA) patients aged 15-24 years [1]. Many cancers that arise in AYA patients, including GCTs, are defined as "rare" because they are relatively infrequent during early childhood and older adulthood [2]. Consequently, the clinical care, clinical trials, and biological study of these cancers have not progressed synchronously with common childhood or adult cancers, and gains in overall survival (OS) for this age group are only half those in either younger or older patients [3]. We reflect on how to address this lack of progress, using as our lens our own experience in creating an international, cross-disciplinary remodeling of the clinical trial development for GCTs to overcome the barriers created by the structure of conventional medical practice.

\section{Reframing the Meaning of "Rare"}

GCTs constitute only $4 \%$ of all pediatric cancer cases diagnosed annually but rank as the third most common AYA cancer after lymphoma and carcinomas and account for up to $20 \%$ of cancer in the male AYA population [4-7]. However, the clinical care for GCTs in AYAs is divided between multiple medical and surgical disciplines (pediatric oncology, gynecology, urology, and medical oncology), and each discipline continues to recognize GCTs as a minor component of their practice. If one were to use neither an adult nor a pediatric frame but rather an AYA frame of patients aged 15-24 years, rare cancers such as GCT would be described as "common" and many common cancers of pediatrics or adulthood would be rare.

\section{REDEFINING OVERALl SuRVIVAL}

Because more than $90 \%$ of patients treated for a GCT are cured, the quality of survival becomes increasingly important for this group of young patients [8]. Five- or even 10-year survival may not be synonymous with normal life expectancy. Travis et al. have shown that a 20 -year old young man, cured of his testicular (GCT) cancer, faces a $>50 \%$ chance of developing a second malignant neoplasm by age 75 , nearly doubling his standard lifetime risk of $26 \%$ of developing cancer $[9,10]$. Woodward et al. reported a twofold risk of cardiovascular disease among testicular cancer survivors [11]. Cisplatin, a key component of the standard chemotherapy treatment for GCT, is measurable in the serum years after exposure: at autopsy, cisplatin has been detected in every organ of the body [12-15]. Furthermore, cisplatin has been shown to be mutagenic and is considered a human carcinogen [16-19].

Serum levels of cisplatin measured 10 years after testicular cancer treatment predict the severity of persistent neuro- and ototoxicity [20]. We cannot be complacent that we have defined the optimal therapy in the face of such significant late effects of standard therapy.

\section{RE-EMPHASIZING THE IMPORTANCE OF RECRUITMENT OF AYAs to Clinical TRIALS \\ Abundant evidence suggests that health care systems that are active in research have better health outcomes because of the introduction of state-of-the-art and standardized care approaches [21-23]. Ferrari et al. have recommended that the}


inclusion of cancer patients in clinical trials be a benchmark of the optimal standard of care [24]. In both the U.K. and the U.S., low recruitment to clinical trials has been documented for AYA patients, with $2.5 \%$ and $13.8 \%$ of patients aged $15-19$ years in the U.K. the U.S., respectively, and falling to $10.6 \%$ of U.K. patients aged $20-24$ years and $4.6 \%$ of U.S. patients $20-29$ years enrolled in a clinical trial $[25,26]$.

Bleyer et al. described multifactorial reasons for poor recruitment of AYA patients to clinical trials [27]. Some barriers to participation include inadequate trial design, failure to open trials for perceived rare tumors in all centers, and a paucity of age-directed information about trials; however, the authors noted that AYAs have shown willingness to participate when clinical trials are available $[28,29]$. Another important factor may be referral pathways: the National Cancer Intelligence Network England reported that $50 \%$ of AYA patients with a gonadal GCT were not referred into a specialist AYA service [30].

Redefining "RARE" In TERms of Clinical Trial Design The International Germ Cell Consensus Group (IGCCG) devised an algorithm to predict overall survival based on risk assessment at diagnosis. Good risk patients have an expected OS of $>90 \%$, but poor risk patients can expect an OS of $<50 \%$ [31]. In the U.S. in 2014, 8,820 men are projected to be diagnosed with testicular cancer $[32,33]$. Using data from SEER18, which includes data on site of metastasis and diagnostic alpha-fetoprotein levels, we estimated that there are approximately 370 new poor-risk testicular cancer patients per year in the U.S. [34]. Under the auspices of multi-institutional national clinical trial organizations such as the Children's Oncology Group (COG) in the U.S. and Canada and the Children's Cancer and Leukemia Group in the U.K., randomized clinical trials of diseases with far fewer than 500 incident cases per year have been successfully conducted. When national sample size has been insufficient, international collaborations have been organized, such as the osteosarcoma EURAMOS trial [35]. However, adult clinical trials conducted over the last decade aiming to identify a regimen with superior outcomes to bleomycin, etoposide, and cisplatin (BEP) chemotherapy in men with IGCCC poorrisk testis cancer have either failed to achieve sufficient accrual to provide statistically meaningful results (e.g., paclitaxel-BEP [36]) or have not been able to progress from an informative, positive phase II trial to a definitive phase III trial because of a lack of conviction within the testicular cancer community that it would be possible to enroll sufficient number of patients (e.g., carboplatin, bleomycin, vincristine, and cisplatin followed by BEP [37]; accelerated $\operatorname{BEP}[38,39])$. A recent exception, the GETUG 13 trial [40], showed that an intensified approach in poor-risk patients who had inadequate tumor marker decline was superior to standard BEP; however, this trial took 9 years to accrue the 260 patients required to answer the study question. We suggest that designing GCT trials based on expected outcome rather than age and gender would allow the GCT community to answer the common outstanding questions around optimization of therapy more efficiently. We contend that it is time to move beyond trial eligibility that is defined only by which clinical trial organization can "lay claim" to the patient.
Solution: Recognition That OPTIMAl CARE OF AYA Patients Requires a NeW Structure and LANGUAGE Within ONCOLOGY

The approach to AYA cancer care started to change with the recognition of the specific medical and psychological needs of AYA patients. Philanthropic organizations have been at the vanguard of this advocacy. In the U.K., the mission statement of the Teenage Cancer Trust is "We exist to improve the quality of life and chances of survival for the six young people aged between 13 and 24 diagnosed with cancer every day in the UK. We want to make sure every one of them has access to the best possible care and professional support from the point of diagnosis [41]." An analogous sister organization, Teen Cancer America, has recently been founded in the U.S. In its landmark publication, "Improving Outcomes: Guidance for Children and Young People with Cancer," the National Institute for Clinical Excellence, which provides clinical practice and quality guidelines to the National Health Service in the U.K., has decreed that AYA-appropriate services must be provided to every AYA cancer patient [42]. Consequently, well-established national referral pathways have been developed for AYA patients to ensure access to cancer care in specialist AYA treatment centers. Teenage Cancer Trust has supported the development of 27 AYA centers within the U.K. to ensure this goal is achieved.

Besides physical infrastructure, Teenage Cancer Trust and other AYA cancer charities, such as the Katie Walker Cancer Trust (U.K.) and the Bridging the Gap Fund (U.S.), have invested in the intellectual capital required to advance the field. With support from these organizations, the Malignant Germ Cell International Collaborative (MaGIC) brought together U.S. and U.K. national experts in pediatric GCTs who agreed to merge deidentified clinical trial data spanning 25 years of platinumbased therapy to develop a more nuanced risk stratification system that could serve as the basis for future collaborative pediatric clinical trials in GCTs, analogous to the IGCCC. This collaboration intentionally included pediatric oncologists, pathologists, surgeons, statisticians, and basic scientists. The resultant risk stratification overturns previously understood risk factors and prognostication and serves as the basis for the new, jointly designed UK-COG clinical trial (J. Nicholson, C. Rodriguez-Galindo, H. Dang et al., manuscript in preparation).

The more challenging frontier in GCTs was to extend knowledge beyond the artificial barriers of age and gender. MaGIC has successfully engaged the National Cancer Research Institute Testis Clinical Study Group and the US Gynecologic Oncology Group. Investigators from these organizations have contributed their respective AYA clinical trial data to extend risk prognostication from early adolescence through young adulthood. Pivotal benefits of these collaborations will be the ability to carry out correlative translational studies that span age and gender. Of note, the recent collaborative formed between COG and SWOG cites MaGIC as an example of good practice (personal communication, D. Freyer) [43].

\section{ConClusion}

For AYA patients with GCTs, AYA units provide the physical setting. The MaGIC model offers a successful template of collaboration to create those age-appropriate clinical trials. 
The joint clinical trial under development by adult and pediatric cooperative groups in the U.S. and the U.K. will offer AYA patients who are diagnosed with GCTs the opportunity to benefit from centralized expertise in a tumor type that is more common in their age group than at any other time during life. In rare cancers, for which small patient numbers preclude statistically meaningful results in a single country, much less a single institution, targeted philanthropic funding to support cross-disciplinary and international work has provided the necessary "magic" to catalyze the first steps in knowledge expansion. Our recommendation is that cancer organizations, both governmental and charitable, continue to fund this approach for rare AYA tumors. A framework that spans age and gender will provide the scaffolding for a more robust approach to the management of diseases historically splintered by our academic oncologic semantics.

\section{ACKNOWLEDGMENTS}

This research was supported by the Bridging the Gap Fund of the Dana-Farber Cancer Institute, the Katie Walker Cancer Trust, the Teenage Cancer Trust, and the William Guy Forbeck Foundation.

\section{AUTHOR CONTRIBUTIONS}

Conception/Design: Sara J. Stoneham, A. Lindsay Frazier, James C. Nicholson, Matthew Murray, Juliet P. Hale, Carlos Rodriguez-Galindo, Ha Dang,
Thomas Olson, James F. Amatruda, Claire Thornton, G. Suren Arul, Deborah Billmire, Mark Krailo, Dan Stark, Al Covens, Jean Hurteau, David Gershenson Provision of study material or patients: Sara J. Stoneham, A. Lindsay Frazier James C. Nicholson, Juliet P. Hale, Carlos Rodriguez-Galindo, Ha Dang, Thomas Olson, Al Covens, Jean Hurteau, Sally Stenning, David Gershenson

Collection and/or assembly of data: Sara J. Stoneham, A. Lindsay Frazier, James C. Nicholson, Juliet P. Hale, Carlos Rodriguez-Galindo, Ha Dang, Thomas Olson, James F. Amatruda, Claire Thornton, Deborah Billmire, Mark Krailo, Dan Stark, Al Covens, Jean Hurteau, Sally Stenning, David Gershenson

Data analysis and interpretation: Sara J. Stoneham, A. Lindsay Frazier, James C. Nicholson, Matthew Murray, Juliet P. Hale, Carlos Rodriguez-Galindo, Thomas Olson, James F. Amatruda, Claire Thornton, G. Suren Arul, Deborah Billmire, Mark Krailo, Dan Stark, Al Covens, Jean Hurteau, Sally Stenning, David Gershenson

Manuscript writing: Sara J. Stoneham, A. Lindsay Frazier, James C. Nicholson, Matthew Murray, Juliet P. Hale, Carlos Rodriguez-Galindo, Ha Dang, Thomas Olson, James F. Amatruda, Claire Thornton, G. Suren Arul, Deborah Billmire, Mark Krailo, Dan Stark, Al Covens, Jean Hurteau, Sally Stenning, David Gershenson

Final approval of manuscript: Sara J. Stoneham, A. Lindsay Frazier, James C. Nicholson, Matthew Murray, Juliet P. Hale, Carlos Rodriguez-Galindo, Ha Dang, Thomas Olson, James F. Amatruda, Claire Thornton, G. Suren Arul, Deborah Billmire, Mark Krailo, Dan Stark, Al Covens, Jean Hurteau, Sally Stenning, David Gershenson

\section{Disclosures}

David Gershenson: American Board of Obstetrics and Gynecology, Asian Society of Gynecologic Oncology, Washington University, Elsevier, UpToDate $(\mathrm{H})$; Johnson \& Johnson, Procter \& Gamble (OI); Carlos Rodriguez-Galindo: Novimmune (C/A). The other authors indicated no financial relationships.

(C/A) Consulting/advisory relationship; (RF) Research funding; (E) Employment; (ET) Expert testimony; (H) Honoraria received; (OI) Ownership interests; (IP) Intellectual property rights/ inventor/patent holder; (SAB) Scientific advisory board

\section{REFERENCES}

1. Rates for 15 to 24 year olds in England. http:// www.nwcis.nhs.uk/tya/tya-incidence/incidencerates-15-24.aspx

2. Surveillance of rare cancers in Europe. Available at http://www.rarecare.eu. Accessed November 26 2013.

3. Smith MA, Seibel NL, Altekruse SF et al. Outcomes for children and adolescents with cancer: Challenges for the twenty-first century. J Clin Oncol 2010;28:2625-2634.

4. Draper GJ, Kroll ME, Stiller CA. Childhood cancer. Cancer Surv 1994;19-20:493-517.

5. Schneider DT, Calaminus G, Koch S et al. Epidemiologic analysis of 1,442 children and adolescents registered in the German germ cell tumor protocols. Pediatr Blood Cancer 2004;42:169-175

6. Poynter JN, Amatruda JF, Ross JA. Trends in incidence and survival of pediatric and adolescent patients with germ cell tumors in the United States, 1975 to 2006. Cancer 2010;116:4882-4891.

7. Arora RS, Alston RD, Eden TO et al. Comparative incidence patterns and trends of gonadal and extragonadal germ cell tumors in England, 1979 to 2003. Cancer 2012;118:4290-4297.

8. Geraci M, Birch JM, Alston RD et al. Cancer mortality in 13 to 29-year-olds in England and Wales, 1981-2005. Br J Cancer 2007;97:1588-1594.

9. Travis LB, Fosså SD, Schonfeld SJ et al. Second cancers among 40,576 testicular cancer patients: Focus on long-term survivors. J Natl Cancer Inst 2005;97:1354-1365.

10. Fung C, Fossa SD, Milano MT et al. Solid tumors after chemotherapy or surgery for testicular nonseminoma: A population-based study. J Clin Oncol 2013;31:3807-3814.

11. Woodward E, Jessop M, Glaser A et al. Late effects in survivors of teenage and young adult cancer: Does age matter? Ann Oncol 2011;22: 2561-2568

12. Tothill $P$, Klys HS, Matheson LM et al. The longterm retention of platinum in human tissues following the administration of cisplatin or carboplatin for cancer chemotherapy. Eur J Cancer 1992; 28A:1358-1361.

13. Gietema JA, Meinardi MT, Messerschmidt J et al. Circulating plasma platinum more than 10 years after cisplatin treatment for testicular cancer Lancet 2000;355:1075-1076.

14. Brouwers EE, Huitema AD, Beijnen JH etal. Longterm platinum retention after treatment with cis platin and oxaliplatin. BMC Clin Pharmacol 2008;8:7.

15. Gerl A, Schierl R. Urinary excretion of platinum in chemotherapy-treated long-term survivors of testicular cancer. Acta Oncol 2000;39:519-522.

16. Greene MH. Is cisplatin a human carcinogen? J Natl Cancer Inst 1992;84:306-312.

17. Travis LB, Holowaty EJ, Bergfeldt K et al. Risk of leukemia after platinum-based chemotherapy for ovarian cancer. N Engl J Med 1999;340:351-357.

18. Travis LB, Andersson M, Gospodarowicz $M$ et al. Treatment-associated leukemia following testicula cancer. J Natl Cancer Inst 2000;92:1165-1171.

19. Vancurová M, Procházková J, Srb V. Monitoring of effects of cis-diamminedichloroplatinum I (Platidiam). Part II. Tests for mutagenic activity in the indicator system of Salmonella typhimurium hisstrains (Ames test). Neoplasma 1985;32:307-312.

20. Sprauten M, Darrah TH, Peterson DR et al. Impact of long-term serum platinum concentrations on neuro- and ototoxicity in cisplatin-treated survivors of testicular cancer. J Clin Oncol 2012;30: 300-307.

21. Karjalainen S, Palva I. Do treatment protocols improve end results? A study of survival of patients with multiple myeloma in Finland. BMJ 1989;299: 1069-1072.

22. Majumdar SR, Roe MT, Peterson ED et al Better outcomes for patients treated at hospitals that participate in clinical trials. Arch Intern Med 2008;168:657-662.

23. Peppercorn JM, Weeks JC, Cook EF et al. Comparison of outcomes in cancer patients treated within and outside clinical trials: Conceptual framework and structured review. Lancet 2004;363:263-270.

24. Ferrari A, Montello $M$, Budd T et al. The challenges of clinical trials for adolescents and young adults with cancer. Pediatr Blood Cancer 2008;50 (Suppl):1101-1104.

25. Fern L, Davies S, Eden Tetal. Rates of inclusion of teenagers and young adults in England into National Cancer Research Network clinical trials: Report from the National Cancer Research Institute (NCRI) Teenage and Young Adult Clinical Studies Development Group. Br J Cancer 2008;99:1967-1974.

26. Bleyer A. Adolescent and young adult (AYA) oncology: The first A. Pediatr Hematol Oncol 2007; 24:325-336.

27. Bleyer A, Budd T, Montello M. Older adolescents and young adults with cancer and clinica trials: Lack of participation and progress in North America. In: Bleyer A, Barr RD, Albritton KH et al. eds. Cancer in Adolescents and Young Adults. Berlin, Germany: Springer Verlag, 2007:71-82.

28. Fern LA, Whelan JS. Recruitment of adolescents and young adults to cancer clinical trialsinternational comparisons, barriers, and implications. Semin Oncol 2010;37:e1-e8.

29. Whelan JS, Fern LA. Poor accrual of teenagers and young adults into clinical trials in the UK. Lancet Oncol 2008;9:306-307.

30. Purkayastha D, O' Hara C, Moran T. Routes to Diagnosis: Investigating the Different Pathways for 
Cancer Referrals in England for Teenagers and Young Adults. London, U.K.: National Cancer Intelligence Network, 2013.

31. International Germ Cell Consensus Classification: A prognostic factor-based staging system for metastatic germ cell cancers. International Germ Cell Cance Collaborative Group. J Clin Oncol 1997;15:594-603.

32. Cancer epidemiology in older adolescents and young adults 15 to 29 years of age, including SEER incidence and survival: 1975-2000. Available at http://seer.cancer.gov/archive/publications/aya/. Accessed March 16, 2014.

33. SEER stat fact sheets: Testis cancer. Available at http://seer.cancer.gov/statfacts/html/testis.html. Accessed March 16, 2014

34. Abrantes F, Ribeiro K, Rodriguez-Galindo C et al. Germ Cell Tumours. Pediatric blood \& cancer special issue: 44th congress of the international society of paediatric oncology, London, United Kingdom, 5th-8th October 2012;59:965-1152.
35. Marina N, Bielack S, Whelan J et al. International collaboration is feasible in trials for rare conditions: The EURAMOS experience. Cancer Treat Res 2009;152:339-353.

36. de Wit R, Skoneczna I, Daugaard G et al. Randomized phase III study comparing paclitaxelbleomycin, etoposide, and cisplatin (BEP) to standard BEP in intermediate-prognosis germ-cell cancer: Intergroup study EORTC 30983. J Clin Oncol 2012;30: 792-799.

37. Christian JA, Huddart RA, Norman A et al. Intensive induction chemotherapy with CBOP/BEP in patients with poor prognosis germ cell tumors. J Clin Oncol 2003;21:871-877.

38. Grimison PS, Thomson DB, Stockler M et al. Accelerated BEP for advanced germ cell tumors. J Clin Oncol 2011;29(suppl).

39. Rimmer Y, Chester J, Joffe J et al. Accelerated BEP:A phase I trial of dose-dense BEP for intermediate and poor prognosis metastatic germ cell tumour. $\mathrm{Br} \mathrm{J}$ Cancer 2011;105:766-772.

40. Fizazi K, Pagliaro LC, Flechon A et al. A phase III trial of personalized chemotherapy based on serum tumor marker decline in poor-prognosis germ-cell tumors: Results of GETUG 13. J Clin Oncol 2013; 31(suppl):LBA4500.

41. Teenage Cancer Trust Factsheet. Available at http://www.teenagecancertrust.org/workspace/ documents/Teenage-Cancer-Trust-Factsheet-2012. pdf. Accessed November 5, 2014.

42. National Collaborating Center for Cancer. Guidance for Cancer Services: Improving Outcomes in Children and Young People With Cancer. The Manual. London, U.K.: National Institute for Health and Clinical Excellence, 2005

43. Freyer DR, Felgenhauer J, Perentesis J. Children's Oncology Group's 2013 blueprint for research: Adolescent and young adult oncology. Pediatr Blood Cancer 2013;60:1055-1058.

\section{For Further Reading:}

Stephen B. Riggs, Earl F. Burgess, Kris E. Gaston et al. Postchemotherapy Surgery for Germ Cell Tumors-What Have We Learned in 35 Years? The Oncologist 2014;19:498-506.

\section{Implications for Practice:}

Patients with advanced testicular cancer will often be considered for surgical consolidation following chemotherapy. This review article focuses on the evaluation and role of surgery in treatment of these complex patients. It underscores the selection of patients, vital role of surgery, as well as providing guidance in the use of surveillance as opposed to surgical extirpation. 